\title{
THE ELEMENTS OF THE BASIC PHOTOMETRICAL CIRCLES ON THE SURFACE OF THE SPHERICAL PLANET AND DETERMINATION OF THEIR PLANETOCENTRIC POSITIONS
}

\author{
V.V. Mikhalchuk \\ Odessa National Maritime Academy, \\ Odessa, Ukraine,vmihalchuk@mail.ru
}

\begin{abstract}
Formulae for determination of planetocentric positions of the basic photometrical circles on the surface of the spherical planet (the equator of intensity, an orthographic limb and orthographic terminator) from the elements of these circles specified to conditions of illumination of its visible disk are obtained. The planetocentric positions of the basic photometrical circles on visible disks of terrestrial planets from their physical ephemerides are calculated.
\end{abstract}

Key words: planets, phase, physical ephemerides, planetocentric coordinates.

\section{Introduction}

At photometrical observations of the surface of planets of the Solar system and their natural satellites there is a problem of determination of the position of features of the local albedo on images of their visible disks. For terrestrial planets which shape can be counted as a first approximation spherical, this problem becomes complicated influence of the phase. It is observed when the geometric planetary disk is illuminated by the Sun not completely, i.e. the phase angle $\Phi$ is distinct from zero. Thus the phase of the planet is considered in an orthographic approximation, i.e. it is supposed, that the Sun is the point source of the light, which is infinitely removed from the planet, hence, the orthographic terminator is the boundary of the illuminated part of the visible planetary disk.

At ground-based and near-earth observations it is possible to neglect radius of the planet in comparison with its geocentric distance. In this case we suppose, that the Earth is infinitely removed from the planet, therefore the initial image of the visible planetary disk represents an orthographic projection of its surface to the plane of the sky.

In contributions (Mikhalchuk, 2001b; Mikhalchuk, 2004a; Mikhalchuk, 2004b; Mikhalchuk, 2004c; Mikhalchuk, 2005a; Mikhalchuk, 2005b; Mikhalchuk, 2007a; Mikhalchuk, 2007b; Mikhalchuk, 2008a;
Mikhalchuk, 2008b) the rotating planetocentric coordinate system with a direction of the calculation of the longitude as in planetographic coordinate system RGS (Sveshnikov, 2004) was considered. This coordinate system also is applied in the present contribution. The base plane of this system is the plane of the equator of the planet, and the position of each point of planet's surface is set by means of the planetocentric latitude $b$ and longitude $l$. At ground-based and near-earth observations of the planet its visible disk is observed in the orthographic projection, therefore the given coordinate system is observed also in the orthographic projection.

In contributions (Mikhalchuk, 2004a; Mikhalchuk, 2004b; Mikhalchuk, 2004c; Mikhalchuk, 2005a; Mikhalchuk, 2005b; Mikhalchuk, 2007a; Mikhalchuk, 2007b; Mikhalchuk, 2008a; Mikhalchuk, 2008b; Mikhalchuk, 2008c) for determination of planetocentric coordinates of the albedo features the auxiliary coordinate system, which also can be applied for photometrical observations, is entered. She the same, as photometric coordinate system, is nonrotating and is linked to equator of intensity, but differs from it by choice of the prime photometric meridian and the direction of the calculation of longitudes.

The position of some photometrical circles on the surface of the spherical planet in an orthographic approximation - the basic photometric meridians in the auxiliary coordinate system, their properties and connection with conditions of illumination of the planet were probed in contributions (Mikhalchuk, 2008b; Mikhalchuk, 2008c). It is shown, as conditions of illumination of the planet determine the shape of the illuminated part of its visible disk and the position of this part on the visible disk. However for binding the illuminated part of the visible planetary disk to the features of the local albedo observed on its surface, it is necessary to attract the planetocentric coordinate system. In turn, the conditions of illumination of the planet set positions of the basic photometrical circles on its surface in the planetocentric coordinate system 
what are the following great circles: equator of intensity, an orthographic terminator and an orthographic limb.

Thus, there is a necessity for a realization a transition from the auxiliary coordinate system to the planetocentric coordinate system for determination of positions of the basic photometrical circles on the surface of the planet. The purpose of the present contribution is determination of elements of the basic photometrical circles, which single-valued setting their position in the planetocentric coordinate system, and deriving of the expressions bundling these elements with conditions of illumination of the planet.

\section{The Basic Photometrical Circles on the Surface of the Planet}

The basic photometrical circles are always clearly designated on the visible planetary disk owing to character of her illumination intensity. They determine the shape of an illuminated part of the visible disk and her position on the visible disk. The shape of an illuminated part of the visible planetary disc is determined by the phase, i.e. depends on the phase angle. The position of an illuminated part on the visible planetary disk relative to the circle of declinations is determined by an angle $Q$, which is the angle of the position of the point of the least illumination of the disk on the geocentric celestial sphere, and relative to the projection of the planet's axis of rotation to the geocentric picture plane is determined by an value $P-Q$, where $P$ is the angle of the position of the planet's axis of rotation on the geocentric celestial sphere.

Let's consider the basic photometrical circles on surface of the spherical planet and their geometric characteristics in the planetocentric coordinate system. Let the visible disk of the spherical planet illuminated by the Sun under the arbitrary phase angle $\Phi$ (Figure 1). Let $A$ is the north pole of the planet and $B$ is the north orthographic cusp of the disk. The equator of the planet is intersected with its orthographic limb in points $G$ and $G^{\prime}$. The basic points of the diameter of intensity: $O$ is the geometric center of the planetary disk (the subterral point), $E$ is the subsolar point (the pole of illumination), $L$ is the pole of phase, $F$ is the point of the least illumination of the disk and $T$ is the visible center of orthographic terminator.

The basic photometrical circles on the surface of the visible planetary disk: equator of intensity, an orthographic terminator and an orthographic limb. All of them are the great circles having the following geometrical meaning:

- for the observer, which is on equator of intensity, centers of the Earth and the Sun are always located on one vertical;

- for the observer, which is on an orthographic ter-

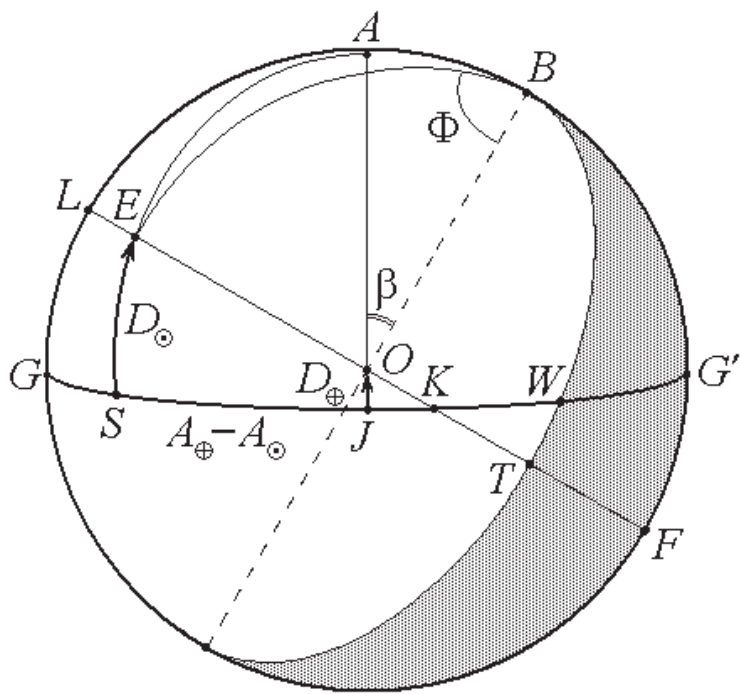

Figure 1: The basic photometrical circles on the visible disk of the spherical planet

minator, the center of the Sun always is in a horizon plane;

- for the observer, which is on an orthographic limb, the center of the Earth always is in a horizon plane.

As the base plane of the planetocentric coordinate system is the plane of equator of the planet planetocentric positions of all remaining photometrical circles should be determined concerning this plane. In the planetocentric coordinate system the basic reference point is the subterral point having planetocentric coordinates $l_{p}$ and $b_{p}$, where $l_{p}$ is longitude of the central meridian passing through the center of the geometric disk is measured from some zero meridian westward of the planet; $b_{p}$ is the latitude of the center of the geometric disk is equal to the planetocentric declination of the Earth $D_{\oplus}$. The position of each photometrical circle in this coordinate system is single-valued set by two elements: the planetocentric longitude $l_{\Omega}$ of his ascending node on equator of the planet and the inclination $i$ of his plane to the plane of the planet's equator. The ascending node of the photometrical circle on equator of the planet is meant as the point of his intersection with equator of the planet $(b=0)$, in the neighbourhood of that for any point of this circle carry out condition $\frac{d b}{d l}>0$. 
3. The connection of planetocentric positions of the basic photometrical circles on the surface of the planet with conditions of its illumination

The equation of the photometrical circle in the planetocentric coordinate system has the form:

$$
\tan b=\tan i \sin \left(l-l_{\Omega}\right) .
$$

Let $P$ and $Q$ are angles of the position of the planet's axis of rotation and the point of the least illumination of the disk on geocentric celestial sphere accordingly (Sveshnikov, 2004). We shall consider angle $\beta$ between the projection of the axis of rotation of the planet on the plane of the sky and the line of cusps (Figure 1), connected with angles $P$ and $Q$ by the following expression (Mikhalchuk, 2004a):

$$
\beta=\mp 90^{\circ}+(P-Q)
$$

The choice of a sign in the formula (2) is carried out by following rule (Mikhalchuk, 2004a): the upper sign concerns to the case, when $\sin (P-Q)>0$, and the lower sign - when $\sin (P-Q)<0$. This convention of signs is spread and further, to all subsequent expressions.

Then, using auxiliary angle $\beta$, it is possible to show, that

$$
l_{\Omega}=l_{p}+u
$$

where the angle $u$ and the inclination $i$ of the plane of the photometrical circle to the plane of equator of the planet are determined from following expressions.

For the equator of intensity

$$
\cos i=\cos D_{\oplus}|\sin (P-Q)| .
$$

The point $K$ of intersection of equator of intensity with equator of the planet, is located on its visible disk, will be called as the defining point of the equator of intensity. It is possible to show, that its planetocentric longitude $l_{K}$ is expressed by the formula

$$
\tan \left(l_{K}-l_{p}\right)=\sin D_{\oplus} \tan (P-Q) .
$$

The point $K$ can prove to be as the ascending node of the equator of intensity on equator of the planet and as the descending node. To find longitude of the ascending node of the equator of intensity by formula (3), it is necessary to make use of expression (5), supposing what $u=l_{K}-l_{p}$, if $\tan (P-Q)<0$. If $\tan (P-Q)>0$ then the found value of the angle $u$ ought to be changed on $180^{\circ}$.

For the orthographic terminator

$$
i=90^{\circ}-\left|D_{\odot}\right|,
$$

where $D_{\odot}$ is the planetocentric declination of the Sun.
The point $W$ of intersection of orthographic terminator with equator of the planet, is located on its visible disk, will be called as the defining point of the orthographic terminator. It is possible to show, that its planetocentric longitude $l_{W}$ is expressed by the formula

$$
l_{W}-l_{p}=\left(A_{\oplus}-A_{\odot}\right) \mp 90^{\circ},
$$

where $A_{\oplus}-A_{\odot}$ is the difference of planetocentric right ascensions of the Earth and the Sun accordingly.

The point $W$ can prove to be as the ascending node of the orthographic terminator on equator of the planet and as the descending node. To find longitude of the ascending node of the orthographic terminator by formula (3), it is necessary to make use of expression (7), supposing what $u=l_{W}-l_{p}$, if $D_{\odot}>0$. If $D_{\odot}<0$ then the found value of the angle $u$ ought to be changed on $180^{\circ}$.

For the orthographic limb

$$
\begin{gathered}
i=90^{\circ}-\left|D_{\oplus}\right| . \\
u=90^{\circ} .
\end{gathered}
$$

In order to remove indeterminacy in calculation of longitude of the ascending node of the orthographic limb on equator of the planet, at $D_{\oplus}<0$ the value of the angle $u$, found of the formula (9), ought to be changed on $180^{\circ}$.

Knowing elements $l_{\Omega}$ and $i$ of the basic photometrical circles, it is possible to determine single-valued the planetocentric position of each of them on the surface of the planet, and from the equation (1) to find the planetocentric latitude $b$ of his any point for the given planetocentric longitude $l$.

4. The planetocentric positions of the basic photometrical circles on the surface of the terrestrial planets

Let's consider the example of determination of planetocentric positions of the basic photometrical circles on the surface of the Mercury, Venus and Mars from their physical ephemerides on $0^{\mathrm{h}} \mathrm{UT}$ on January 8, 2004 (Glebova, 2003), which are listed in Table 1.

The planetocentric declination of the Sun for Mercury is equal zero, hence, the Sun is located in the plane of his equator. Then from the formula (6) follows, that for an orthographic terminator of Mercury $i=90^{\circ}$.

The elements of the basic photometrical circles, which are calculated by the formulae (3), (4), (6), (8) and (9), are submitted in Table 2.

The results of calculation, which are listed in Table 2 , are completely accorded with the physical ephemerides of planets from Table 1 . 
Table 1: The physical ephemerides of planets on $0^{\mathrm{h}}$ UT January 8, 2004

\begin{tabular}{cccccccc}
\hline Planet & $P$ & $D_{\oplus}$ & $A_{\oplus}-A_{\odot}$ & $D_{\odot}$ & $\Phi$ & $Q$ & $l_{p}$ \\
\hline Mercury & $7^{\circ} .08$ & $-7^{\circ} .13$ & $+105^{\circ} .32$ & $0^{\circ} .00$ & $105^{\circ} .2$ & $279^{\circ} .03$ & $261^{\circ} .81$ \\
Venus & 342.18 & +1.29 & -50.70 & +1.88 & 50.68 & 73.55 & 352.94 \\
Mars & 327.12 & -25.12 & -41.84 & -12.38 & 41.42 & 67.12 & 154.90 \\
\hline
\end{tabular}

Table 2: The elements of the basic photometrical circles of planets on $0^{\mathrm{h}}$ UT January 8, 2004

\begin{tabular}{ccccccc}
\hline Planet & \multicolumn{2}{c}{ Equator of intensity } & \multicolumn{2}{c}{ Terminator } & \multicolumn{2}{c}{ Limb } \\
& $i$ & $l_{\Omega}$ & $i$ & $l_{\Omega}$ & $i$ & $l_{\Omega}$ \\
\hline Mercury & $7^{\circ} .39$ & $7^{\circ} .15$ & $90^{\circ}$ & $277^{\circ} .13$ & $82^{\circ} .87$ & $171^{\circ} .81$ \\
Venus & 1.88 & 216.21 & 88.12 & 32.24 & 88.71 & 82.94 \\
Mars & 26.92 & 267.46 & 77.62 & 23.06 & 64.88 & 64.90 \\
\hline
\end{tabular}

The elements of the basic photometrical circles of planets allow compiling the equations of these circles in the planetocentric coordinate system, to build them on the surface of the planet and to determine the planetocentric coordinates of each point of the given circle.

By means of the program's batch (Mikhalchuk, 2001a) computer simulation of projection of the basic photometrical circles of planets on the plane of the sky was produced. The obtained computer models completely are accorded with the images of circles, which built on their elements from Table 2 .

\section{Conclusion}

The main results obtained in this contribution:

1. The basic photometrical circles on the surface of the spherical planet are considered. The geometric meaning of these circles for the observer, which is on its, is shown.

2. The formulae, which allow calculating the elements of the basic photometrical circles on the surface of the spherical planet, are obtained.

3. The elements of the basic photometrical circles on the planet's surface depend on its orientation concerning the Earth and the Sun, and also are determined by conditions of illumination of the visible planetary disk.

Acknowledgements. The authors are thankful to anybody who has read this contribution to the end.

\section{References}

Glebova N.I., Ed.: 2003, Astronomical Year-Book for
2004. St. Petersburg: IPA RAN.

Mikhalchuk V.V.: 2001a, Odessa Astron. Publ., 14, 261-264.

Mikhalchuk V.V.: 2001b, Astron. Vestn., 35, N 1, 89-96.

Mikhalchuk V.V.: 2004a, Kinematics and Physics of Celestial Bodies, 20, N 1, 76-92.

Mikhalchuk V.V.: 2004b, Abstract book of the Conf. VAK-2004 "Horizons of the Universe". Transact. SAI, 75, Moscow (Russia), 234.

Mikhalchuk V.V.: 2004c, Odessa Astron. Publ., 17, 54-57.

Mikhalchuk V.V.: 2005a, Kinematics and Physics of Celestial Bodies, Suppl. Ser., 5, 557-560.

Mikhalchuk V.V.: 2005b, Proc. VIII Conf. of Astron. Soc. and Int. Symp. "Astronomy-2005: Situation and Development Prospects". Transact. SAI, 78, Moscow (Russia), 13.

Mikhalchuk V.V.: 2007a, Odessa Astron. Publ., 20, Part 2, 76-80.

Mikhalchuk V.V.: 2007b, Transact. of the All-Russia Astronomical Conf. VAK-200\%. Kazan (Russia), 49-51.

Mikhalchuk V.V.: 2008a, Radiophys. and radioastron., 13, N 3, 134-139.

Mikhalchuk V.V.: 2008b, Program and Abstracts of the VIII-th G.Gamow's Odessa Astronomical Summer School "Astronomy and beyond: Astrophysics, Radioastronomy, Cosmology and Astrobiology". Odessa (Ukraine), 21.

Mikhalchuk V.V.: 2008c, Abstract Book of the International Conf. "The Solar System Bodies: from Optics to Geology". Kharkiv (Ukraine), 89-90.

Sveshnikov M.L.: 2004, Tr. IPA RAN, 10: Ephemeris Astronomy /ed. Finkel'shtein A.M., St. Petersburg: IPA RAN, 349-375. 